University of Wollongong

Research Online

Faculty of Social Sciences - Papers (Archive) Faculty of Arts, Social Sciences \& Humanities

2012

Acceptance and Commitment Therapy (ACT) for Psychological Adjustment after Traumatic Brain Injury: Reporting the Protocol for a Randomised Controlled Trial

\author{
Diane L. Whiting \\ University Of Wollongong \\ Grahame K. Simpson \\ University Of Sydney \\ Hamish J. Mcleod \\ University Of Glasgow \\ Frank P. Deane \\ University of Wollongong, fdeane@uow.edu.au \\ Joseph Ciarrochi \\ University of Wollongong, joec@uow.edu.au
}

Follow this and additional works at: https://ro.uow.edu.au/sspapers

Part of the Education Commons, and the Social and Behavioral Sciences Commons

Research Online is the open access institutional repository for the University of Wollongong. For further information contact the UOW Library: research-pubs@uow.edu.au 


\title{
Acceptance and Commitment Therapy (ACT) for Psychological Adjustment after Traumatic Brain Injury: Reporting the Protocol for a Randomised Controlled Trial
}

\begin{abstract}
Following a severe traumatic brain injury (TBI) there is a complex presentation of psychological symptoms which may impact on recovery. Validated treatments addressing these symptoms for this group of people are limited. This article reports on the protocol for a single-centre, two-armed, Phase II Randomised Control Trial (RCT) to address the adjustment process following a severe TBI. Participants will be recruited from Liverpool Brain Injury Rehabilitation Unit and randomly allocated to one of two groups, Acceptance and Commitment Therapy (ACT) or an active control (Befriending). The active treatment group utilises the six core processes of ACT with the intention of increasing participation and psychological flexibility and reducing psychological distress. A number of primary and secondary outcome measures, administered at assessment, post-treatment and 1-month follow-up, will be used to assess clinical outcomes. The publication of the protocol before the trial results are available addresses fidelity criterion (intervention design) for RCTs. This ensures transparency in the RCT and that it meets the guidelines according to the CONSORT statement. The protocol has also been registered on the Australian New Zealand Clinical Trials Registry ACTRN12610000851066.
\end{abstract}

\section{Keywords}

after, injury, therapy, brain, adjustment, act, psychological, traumatic, acceptance, protocol, randomised, controlled, trial, commitment, reporting

\section{Disciplines}

Education | Social and Behavioral Sciences

\section{Publication Details}

Whiting, D. L., Simpson, G. K., Mcleod, H. J., Deane, F. P. \& Ciarrochi, J. (2012). Acceptance and Commitment Therapy (ACT) for Psychological Adjustment after Traumatic Brain Injury: Reporting the Protocol for a Randomised Controlled Trial. Brain Impairment, 13 (3), 360-376. 


\section{Acceptance and Commitment Therapy (ACT) for psychological adjustment after traumatic brain injury: Reporting the protocol for a Randomised Controlled Trial}

Authors:

Whiting $\mathrm{DL}^{1,2}$, Simpson $\mathrm{GK}^{1,3}$, McLeod $\mathrm{HJ}^{4}$, Deane $\mathrm{FP}^{2}$, Ciarrochi $\mathrm{J}^{5}$

Affiliations:

1. $\quad$ Liverpool Brain Injury Rehabilitation Unit, Liverpool Hospital, Sydney

2. School of Psychology, University of Wollongong, Australia

3. Rehabilitation Studies Unit, University of Sydney, Australia

4. Institute of Health and Wellbeing, University of Glasgow, Scotland

5. School of Social Sciences and Psychology, University of Western Sydney, Australia

Keywords: Acceptance and Commitment Therapy, traumatic brain injury, psychological distress, randomised controlled trial, participation

Correspondence to:

Diane Whiting

Senior Clinical Psychologist

Brain Injury Rehabilitation Unit

Liverpool Hospital

Locked Bag 7103

Liverpool BC NSW 1871 Australia

Phone: 61298285495

Fax: 61298285497

E-mail: diane.whiting@sswahs.nsw.gov.au 


\begin{abstract}
Following a severe traumatic brain injury (TBI) there is a complex presentation of psychological symptoms which may impact on recovery. Validated treatments addressing these symptoms for this group of people are limited. This article reports on the protocol for a single centre, two armed, Phase II Randomised Control Trial (RCT) to address the adjustment process following a severe TBI. Participants will be recruited from Liverpool Brain Injury Rehabilitation Unit and randomly allocated to one of two groups, Acceptance and Commitment Therapy (ACT) or an active control (Befriending). The active treatment group utilises the six core processes of ACT with the intention of increasing participation and psychological flexibility and reducing psychological distress. A number of primary and secondary outcome measures, administered at assessment, post treatment and one month follow up, will be used to assess clinical outcomes. The publication of the protocol before the trial results are available addresses fidelity criterion (intervention design) for RCTs. This ensures transparency in the RCT and that it meets the guidelines according to the CONSORT statement. The protocol has also been registered on the Australian New Zealand Clinical Trials Registry ACTRN12610000851066.
\end{abstract}




\section{Distress following traumatic brain injury}

The impact of traumatic brain injury (TBI) often results in a complex presentation of psychological symptoms and associated distress. These symptoms can encompass anxiety, depression, anger, grief and traumatic stress. The resulting psychological distress can be linked to the traumatic event causing the injury, to loss of functioning or a combination of both these factors. It has been proposed that chronic inflammatory processes in the brain may be an underlying mechanism that contributes to the development of depression and stress responses and inhibits the process of repair (Hoffman \& Harrison, 2009). Furthermore, the ability to cope with these symptoms is often complicated by the cognitive changes brought about by the brain injury, by psychosocial factors, and by premorbid coping styles (Ponsford, Sloan, \& Snow, 1995).

High levels of psychological distress after a TBI have been well documented. A recent systematic review concluded that there was a 33\% prevalence rate of depression from one year and later post-injury (Guillamondegui et al., 2011). Hibbard et al., (1998) found that rates for anxiety symptoms reported in the literature ranged between $18 \%$ and $60 \%$. Not only are psychological symptoms evident but there can also be disruption in the way people perceive their sense of self (Myles, 2004). People with a TBI may no longer have a stable sense of "who they are" and tend to view the self more negatively after a TBI (Carroll \& Coetzer, 2011). These psychological symptoms can also co-present with behavioural problems such as verbal and physical aggression (Baguley, Cooper, \& Felmingham, 2006; Rao et al., 2009).

All of these factors contribute to a complex adjustment process which influences the person's ability to (i) engage in many aspects of their rehabilitation and (ii) become involved in meaningful activities (Fleming et al., 2011). Lower levels of depression have been associated with increased participation in social and recreational activities after a brain injury (Brown, Gordon, \& Spielman, 2003). Therefore, timely intervention for these adjustment difficulties may lead to improved 
participation in rehabilitation and engagement in their life such that clients become better able to accept their changes and move on with their lives.

\section{Psychological treatments for emotional/behavioural adjustment after TBI}

Early psychological interventions in the treatment of TBI predominantly drew upon learning/behaviour theory and these approaches continue to play an important role, particularly in the management of challenging behaviours (Schlund \& Pace, 1999; Wood \& Alderman, 2011). The suitability of cognitive approaches (e.g., cognitive behavioural therapy, motivational interviewing and problem-solving therapy) has also been investigated, particularly for the treatment of psychological distress. Cognitive Behaviour Therapy (CBT) has been considered particularly applicable for those with a TBI because of the structured nature of the treatment and the ability to adapt the therapy to individual requirements (Khan-Bourne \& Brown, 2003). These adaptions can include the use of written notes during sessions, undertaking one task at a time, using repetition to ensure new concepts are learned and breaking down tasks into smaller parts (Hibbard, Rendon, Charatz, \& Kothera, 2005).

Programs using CBT have proven to be effective in treating a range of post TBI psychological problems including anger (Medd \& Tate, 2000), anxiety (Hsieh, Ponsford, Wong, Schonberger et al., 2012), coping skills (Anson \& Ponsford, 2006), hopelessness (Simpson, Tate, Whiting, \& Cotter, 2011) and social anxiety (Hodgson, McDonald, Tate, \& Gertler, 2005). Despite these promising developments, the number of high quality studies employing randomized controlled designs (RCTs) to evaluate psychological treatments for people with severe TBI is sparse. Systematic reviews have identified no cognitive interventions rated as Class 1 for depression (Fann, Hart, \& Schomer, 2009) or anxiety (Soo \& Tate, 2009). The one Class 1 study identified by Soo and Tate (2009) treated an intervention for Acute Stress Disorder among people with mild TBI (Bryant, Moulds, Guthrie, \& Nixon, 2003). A subsequent RCT for the treatment of anxiety has just 
been published (Hsieh, Ponsford, Wong, Schönberger et al., 2012) and holds the promise of being rated highly for its robust methodology. Only one RCT has been identified for the treatment of anger (Medd \& Tate, 2000), and rated as Class 1 in one review (Ylvisaker et al., 2007) but Class 2 due to the small sample size in a second review (Cattelani, Zettin, \& Zoccolotti, 2010). Overall, there are only a handful of robust psychological treatment studies of post-TBI adjustment that can be relied upon to guide decisions about the best approach to this significant problem. There is an urgent need for more high quality studies in this area.

In seeking to expand this limited evidence base, there are also reasons to question whether adapted forms of CBT represent the best treatment approach. One potential limitation of CBT for post-TBI adjustment is its emphasis on thought challenging (Kinney, 2001). Cognitive impairments and related problems with self-awareness may make challenging unhelpful thought processes very difficult for people with TBI (Sherer et al., 1998). Similarly, problems with divided attention may reduce the capacity to simultaneously hold thoughts in mind while seeking alternative or more helpful ways of thinking (Blanchet, Paradis-Giroux, Pépin, \& Mckerral, 2009). The cognitive inflexibility often seen after a TBI may also reduce the capacity to shift to more helpful or adaptive thinking patterns (Heled, Hoofien, Margalit, Natovich, \& Agranov, 2012). Given these concerns, newer forms of cognitive therapy may also play a significant role in treating the problems of adjustment to TBI.

\section{Acceptance and Commitment Therapy (ACT)}

Acceptance and Commitment Therapy (ACT) is one of the 'third-wave' of behavioural therapies, with a focus on changing one's relationship with internal experiences (thoughts, feelings, memories and physical sensations) rather than on directly changing the content of these experiences. The key premise of ACT is to teach people to be able to have internal experiences, in a mindful and nonjudgmental way, and still engage in effective action. The ACT model is comprised of six core 
processes which form a hexaflex indicating that all components are presumed to be interlinked (Hayes, Strosahl, \& Wilson, 2003). The therapist can elect to work on any of the components of the model at any stage of the therapy process or these components can be combined and presented at the same time. In this respect use of the hexaflex components is not seen as linear.

The six core processes which configure the hexaflex and guide the process of developing psychological flexibility include cognitive defusion, acceptance, contact with the present moment, self as context, values and committed action (Hayes et al., 2003). Cognitive defusion is the process of creating some distance or separation from distressing thoughts, emotions or experiences. Acceptance is the opening up and making room for these internal experiences so there is no longer an ongoing struggle. Contact with the present moment is being in the here and now, consciously connecting with is happening in that moment. Self as context or the observing self seeks to demonstrate that a component of us is always the same, regardless of what is changing with regard to our feelings or experiences. Values are what guide our behaviour and are unique and personally relevant to each individual. They assist in setting goals which is the committed action component of the hexaflex.

\section{Effectiveness of ACT in chronic health conditions}

Prior research using ACT with other chronic health conditions suggests it may be promising for use with people who have a TBI. The research into ACT and chronic pain has been the most extensive. A recent review identified 11 studies between 2004 and 2009 which used ACT with this population, of which three were RCTs (Ruiz, 2010). More recently a meta-analysis of acceptance based interventions for chronic pain found small to moderate effect sizes for reducing the pain experience and concluded that ACT maybe a good alternative to CBT with this population (Veehof, Oskam, Schreurs, \& Bohlmeijer, 2011). In other chronic health conditions, ACT has also been found to be efficacious in improving coping with diabetes (Gregg, Callaghan, Hayes, \& Glenn- 
Lawson, 2007), in reducing the impact of tinnitus (Hesser, Westin, Hayes, \& Andersson, 2009) and maintaining activity levels despite no change in pain levels (Dahl, Wilson, \& Nilsson, 2004). Given the growing evidence for the efficacy of ACT among other clinical populations, the possible value of ACT in facilitating the process of adjustment and reducing distress after TBI warrants investigation.

\section{Rationale for using ACT with TBI population}

Support for ACT after brain injury has been discussed in two recent reviews (Kangas \& McDonald, 2011; Soo, Tate, \& Lane-Brown, 2011). Kangas and McDonald (2011) considered the implementation of ACT in the context of mild to moderate acquired brain injury (ABI). They proposed that ACT may assist people after an ABI in moving forward with their lives by accepting their cognitive and physical changes. They also made a number of recommendations for the design of a treatment programme. In a review of the applicability of ACT for treatment of anxiety after ABI, Soo and colleagues (2011) recommended that the behavioural based components of ACT such as values and committed action should be emphasised but modifications for the more cognitive components such as defusion may be enhanced by using concrete examples, repetition and use of written aids. The current study will investigate how the core processes within ACT can be operationalised to accommodate the cognitive impairments evident after a TBI and provide an alternative to cognitive strategies such as thought challenging. In addition, the treatment program will seek to improve psychological flexibility and encourage committed action in accordance with individual values.

Following a brain injury, there is often a protracted period of recovery, cognitive improvements can take years to achieve or, the person can be left with persistent cognitive and emotional impairments (deGuise et al., 2008). Thoughts such as "my brain injury stops me doing what I want to do" or "my life is over because of my brain injury” can emerge as part of these 
persistent impairments. Challenging these thoughts using standard CBT techniques may be particularly difficult when the person is cognitively impaired (Kinney, 2001) and studies using cognitive restructuring strategies report that many patients have difficulty implementing such strategies (Anson \& Ponsford, 2006). The ACT approach reduces this problem by focussing on mindfulness-based approaches that do not require the client to engage in reasoning or evaluating evidence. These approaches help the client make space for difficult experience (e.g., not avoid it), to notice such experience with openness and curiosity, and to become less reactive to such experience. Clients learn to mindfully observe a thought like "I am stupid" and yet not let that thought dominate how they behave. For example, an ACT practitioner might assist the patient to "make space” for unpleasant thoughts by using concrete strategies such as "physicalising” the thought (Hayes et al., 2003). Another potential advantage of the use of experiential role plays and visual metaphors as a part of ACT, is that it makes the therapy less reliant on verbal means of expression. The metaphors can be tailored to the client's background and may also use pictorial representations. Information is therefore delivered in more than one modality which is desirable in people experiencing difficulties in processing information from therapeutic counselling sessions (Simpson et al., 2011).

After a TBI, people need to define themselves in conjunction with both their cognitive and physical limitations (Whitehouse, 1994). This may inhibit them from developing new behaviours and reconnecting with life. ACT helps clients to let go of limited senses of self and to develop patterns of adaptive behaviour. Thus the acceptance component of ACT may be helpful in facilitating adjustment to unpleasant physical changes and the value clarification and commitment component can support behavioural activation in the presence of those changes. Perhaps most importantly, ACT aims to promote greater participation in activities congruent with a person's values and this is an under-examined therapeutic need in people who have suffered a TBI. The concept of addressing a person's values in order to develop a unique meaning for them after their 
injury was proposed before ACT was developed (Wright, 1960). Values create an opportunity to define and personalise goals in order to make them more client focussed and relevant to the client. The use of client centred goals is a well-researched and validated approach to ensure effective rehabilitation post TBI (Doig, Fleming, Cornwell, \& Kuipers, 2009).

Finally, ACT is also hypothesised to increase psychological flexibility and the ability to persist with values-consistent behaviour in the face of challenges and obstacles. Psychological flexibility maybe related to cognitive flexibility (Chawla \& Ostafin, 2007; Kashdan \& Rottenberg, 2010). As already stated, impairments in cognitive flexibility are common after TBI (Heled et al., 2012) and are often a factor in poor treatment response, resulting in functional difficulties many years after a brain injury (McDonald et al., 2002). We could speculate that ACT may benefit people with TBI by improving both psychological and cognitive flexibility.

\section{Testing the feasibility of ACT in TBI}

Some initial evidence does exist for the benefits of ACT among people with cognitive impairment. An ACT intervention was successfully used to increase participation and adaptive functioning in adolescents and adults (ages 15-59 years) who had acquired their brain injury before the age of 18 years, i.e. paediatric acquired brain injury (Sylvester, 2011). There is also a case study where ACT was used to successfully treat an adolescent with learning disabilities to better manage anxious thoughts and obsessive ruminations (Brown \& Hooper, 2009).

In preparation for the current trial, a feasibility study to evaluate the ACT treatment program was also conducted at the Liverpool BIRU. (Whiting, Simpson, Ciarrochi, \& McLeod, 2012). The study was undertaken with two participants, both young males (20 and 29 years) who were diagnosed with a severe TBI (Posttraumatic Amnesia of 3 and 17 days) and were reporting psychological distress as measured by the Depression Anxiety and Stress Scale-21. They jointly engaged in a seven session, manualised treatment program based on ACT principles. Data were 
analysed and presented as two separate case studies with pre and post-treatment measures of mood, psychological flexibility and participation undertaken, in addition to sessional measures. The primary outcome measure, the Acceptance and Action Questionnaire-Acquired Brain Injury (AAQ$\mathrm{ABI})$, is a measure of psychological flexibility developed to target the types of issues occurring after a brain injury. This was adapted from a measure used in a study with paediatric acquired brain injury (Sylvester, 2011). Other outcome measures included a measure of social participation and psychological distress. Both participants demonstrated improvements in their psychological flexibility with one participant's change being significant $(\mathrm{RCI}=1.98)$. They both demonstrated significant decreases in their level of psychological distress. One participant also reported an increase of 6.9 points on participation, approaching the criterion for significant reliable change (a change of 8 points). In addition to this, both participants achieved goals which had been established in accordance with their identified values indicating further support for the utility of ACT following a severe TBI. The successful outcome from the feasibility study has laid the groundwork for conducting the proposed protocol.

\section{Conducting trials of psychological interventions in TBI: Design issues}

To test the efficacy of ACT among people with TBI, a number of research design issues from previous trials experience helped shape the current protocol. As a starting point, the nature of Phase II and Phase III intervention trials treating the emotional or behavioural sequelae of people with severe TBI are qualitatively different from biomedical trials in which the same terminology is employed. Biomedical Phase II trials test the efficacy of a treatment (answering the question does it work?) and typically involves enrolling 100-300 patients in a pre- and post-test trial. Phase III trials then address the question (is this treatment better than current best practice?) by means of RCTs that may enrol thousands of patients. 
In the field of TBI, the first difference is that Phase II trials often employ a randomized controlled design. In the context of nursing research, Feeley and associates (2009) have argued that the use of pilot RCTs for Phase II trials can act as a precursor, testing acceptability and feasibility of treatment and trial delivery, as well as treatment efficacy. This lays the groundwork for subsequent Phase III trials. Consistent with this argument, the randomised controlled trials evaluating psychological interventions for affective and/or behavioural disturbance among people with severe TBI can be described as Phase II, all having sample sizes of less than 30 participants (Hsieh, Ponsford, Wong, Schonberger et al., 2012; Medd \& Tate, 2000; Simpson et al., 2011). Despite the small samples, the effect sizes for primary outcome variables from these studies has generally been robust (ES 0.89, Medd \& Tate, 2000; ES>1.0, Simpson et al., 2011; ES 0.50, Hsieh et al., 2012).

Another characteristic of the trials is the intensity of intervention. In one of the first systematic reviews of evidence for the efficacy of neurorehabilitation it was concluded that many of the interventions lacked sufficient intensity (Carney et al., 1999). The interventions tested in the trials involved between 12 and 20 hours of therapy, highlighting the level of input that people with severe TBI require to benefit from cognitively-based interventions. This level of intensity is consistent with the recommendations of Kangas and MacDonald (2011) in their discussion about the delivery of an ACT program in TBI. There has also been ongoing debate about the relative merits of individual versus group-based delivery of therapeutic interventions. In a direct comparison of the two modalities it was found that individual therapy was more effective than group therapy in treatment for post-injury impairments of awareness (Ownsworth, Fleming, Shum, Kuipers, \& Strong, 2008). Subsequent research suggests that small groups of two people can also be effective (e.g., Simpson et al., 2011). One benefit of keeping any group small is that it mitigates the risk of a dilution of treatment intensity. 
Systematic reviews of treatment studies for post-TBI affective problems have highlighted the absence of clinically significant threshold levels of the targeted disorders in some studies (Fann et al., 2009; Soo \& Tate, 2009). The most recent trials have employed such thresholds by using cutoff scores on standardised measures (Simpson et al., 2011) or participants meeting clinical criteria for psychiatric disorders (Hsieh, Ponsford, Wong, Schönberger et al., 2012). The current trial therefore needed to clearly define the treatment population.

Another important consideration was the selection of the control condition, and this issue has been canvassed in some detail as it applies to experience-based treatments delivered in the rehabilitation context (Hart, Fann, \& Novack, 2008). An active control condition typically contrasts some other type of standardised intervention to control for the level of therapist attention and degree of treatment accessed. This is stronger than a TAU condition, because TAU conditions often do not result in equivalent levels of intervention, and may in fact involve the provision of limited or no services during the study period (Hart et al, 2008). In addition, a credible active control can help maximise participant engagement in the trial. Despite the value of the active control, the Phase II published trials to date have typically employed TAU or standard care conditions. Bryant et al., (2003) provided one of the few exceptions, having employed an active control (i.e., a non-specific problem-solving program) in their trial for the treatment of acute stress disorder, albeit in a mild TBI sample. One of the challenges in the introduction of active controls is to ensure that they are manualised (Hart et al., 2008; Schulz, Altman, \& Moher, 2010; Whyte \& Hart, 2003)in order to achieve an equivalent level of standardisation across treatment and control.

Whyte and Hart (2003) have also highlighted the problems associated with poor selection of outcome measures in rehabilitation intervention studies. One of the challenges in trialling new treatments can be the limited availability of appropriate outcome measures. In the ACT literature, the desired outcome of treatment is often increased participation and psychological flexibility. Effective measurement of psychological flexibility usually involves developing questionnaires to 
ensure the content is targeted to specific disorders and populations. As ACT has yet to be effectively used in a TBI population, no validated measures currently exist nor has the generic measure of psychological flexibility, the Acceptance and Action Questionnaire-II (AAQ-II) (Bond et al., 2011) been validated on a TBI population. The issues outlined in this section informed the current trial protocol including (i) selection of research design, (ii) sample size, (iii) intensity of therapy, (iv) mode of delivery, (v) use of a diagnostic threshold, (vi) use of an active control, and (vii) selection of process and outcome measures. Furthermore, the protocol was developed in accordance with CONSORT statement (Schulz et al., 2010) as recommended by Tate and Douglas (2011).

\section{Treatment Programs}

As developed and tested in the feasibility pilot study, the ACT treatment program (titled "Act on adjusting after your brain injury”) will incorporate all six core processes of ACT with a focus on each process during each week of the program. Both mindfulness exercises and values work will be woven throughout the program with both these components addressed in more detail during specific sessions. See Table 1 for a summary of the treatment program. The program will use a treatment manual and participants will be given a workbook in which to complete in-session activities and undertake homework tasks.

\section{(Insert Table 1 here)}

\section{Befriending}

The active control will be based on the Befriending protocol (Bendall, Killackey, Jackson, \& Gleeson, 2003). Befriending controls for a number of variables which may confound treatment programs and has been found to have a moderate affect in reducing depressive symptoms and emotional distress in a number of different populations (Mead, Lester, Chew-Graham, Gask, \& 
Bower, 2010). It was found to be a credible control for factors such as enjoyment of therapy, the dropout rate and client expectations against a CBT treatment for early psychosis (Bendall et al., 2006). The goal of the Befriending group was to engage participants in neutrally loaded topics that were of interest to the participants for the same duration as the ACT group. During session one, topics are identified for the following five sessions through a collaborative process. A suggested list of topics will be provided to participants (Table 2) to assist with idea generation. Emotionally loaded topics are avoided and participants are redirected to neutral topics should the conversation move in that direction. One session is dedicated to an outdoor excursion, usually session two, to the local coffee shop. The group is run so that each participant and the therapist talks in turn on their topic and answers questions. This allows the group to remain structured and equal time is delegated for each participant to engage in conversation. They are also given the topics prior to the session which allows for some homework activity and engagement.

(Insert Table 2 here)

\section{Aims and Hypotheses}

The aim of the project is to elucidate the process of change during an ACT therapeutic intervention and explore the therapeutic processes involved in ACT using a time series analysis. The following hypotheses will be tested: (1) ACT treated patients will demonstrate improved psychological flexibility and lower psychological distress after treatment compared to patients receiving the active control treatment (Befriending) (Bendall et al., 2003). (2) Improved psychological flexibility will predict lower levels of psychological distress following treatment. (3) ACT treated patients will demonstrate greater participation in rehabilitation compared to patients receiving Befriending.

\section{Study Design}


A single centre phase II randomised controlled trial will be conducted in accordance with the CONSORT statement (Schulz et al., 2010) (see Figure 1). The intervention will be undertaken at Liverpool Brain Injury Rehabilitation Unit (LBIRU) at Liverpool Hospital and participants recruited from the community outpatient service. The LBIRU provides rehabilitation services to people with a severe TBI (i.e. > 24 hours posttraumatic amnesia) in South-Western Sydney. Ethics approval for the project has been obtained by Sydney South West Area Health Ethics Committee and the Human Research Ethics Committee of the University of Wollongong. The study is being undertaken as part of the requirements for the degree of Doctor of Philosophy by the first author (DLW). The protocol has also been registered on the Australian New Zealand Clinical Trials Registry ACTRN12610000851066.

(insert Figure 1 here)

\section{Participants}

Participants who meet the study inclusion criteria will be recruited over a 2-year period from the LBIRU community outpatient team. The criteria are, (i) sustained an extremely severe traumatic brain injury (Posttraumatic Amnesia; PTA > 1 week; Russell \& Smith, 1961), (ii) injury after the age of 18 years (iii) currently between 18 and 65 years, (iv) have sufficient cognitive capacity and English language skills to complete questionnaires and engage in group discussions (determined by existing neuropsychological assessments and in consultation with staff at the BIRU), (v) report a moderate range or greater on any of the subscales of the Depression Anxiety and Stress Scale-21 (DASS-21; Lovibond \& Lovibond, 1995), and (v) have not have been diagnosed with a severe psychiatric disorder prior to the TBI.

\section{Sample Size}

We aim to recruit 48 participants over a 2-year period from the LBIRU community outpatient team. 
The LBIRU accepts 120 new referrals each year and has an active caseload of more than 400 clients with severe TBI of which approximately $90 \%$ are severe TBI. Average annual referrals to Clinical Psychology from 2009-2010 have been 73 clients. A review of these referrals indicates that approximately $50 \%$ of these clients would meet the study criteria for the study and with an anticipated refusal rate of $20 \%$ a sample size of 48 appears realistic for the specified time period. In addition for a large effect size (.89), and a one-tailed test, a sample size of 48 will provide over .90 power to detect a difference between the two groups.

\section{Intervention Fidelity}

To ensure treatment fidelity, the five domains recommended by Borelli (2011) were followed:

(i) Intervention design has been addressed by registration of the trial protocol followed by this publication of the protocol in a peer-reviewed journal. This allows the study design to be described and independently reviewed; (ii) Training of providers in the ACT intervention will all be undertaken by the first author, who has eight years' experience in TBI and four years as an ACT therapist. Both groups will be implemented by a registered psychologist, experienced in both ACT and TBI. The first author will also receive ACT supervision from a recognised ACT trainer who is part of the research team (JC); (iii) Standardisation in intervention delivery will be enabled by employing a written manual for both treatment conditions. In addition, treatment sessions will be audio recorded and random samples of sessions (25\%) reviewed by trained independent assessors for treatment fidelity. The ACT Core Competency Rating Scale (Hayes \& Strosahl, 2004) and the Befriending fidelity scale (Bendall et al., 2003) will be used to check that the ACT treatment sessions and Befriending intervention are delivered respectively in accordance with the manualised programs; (iv) Fidelity in receipt of intervention is particularly relevant with TBI patients as they are exhibiting cognitive impairment and may struggle with comprehension. Each session will commence with a review of the previous week's session and participants will be given written 
notes of the session and homework tasks to complete. Adherence to and acceptability of homework will be monitored using a homework form which will be completed at the end of each sessions and prior to the next session commencing (available from the first author on request). Sessions will be coordinated to ensure attendance in treatment does not fall below $80 \%$ of all sessions. To maximise attendance, participants will be sent text messages or given reminder phone calls the day prior to the treatment session;(v) Ensuring the treatment is enacted in real life settings will be assessed during session seven. This is a relapse prevention session where feedback will be used to discuss how participants are using the strategies outside the group environment.

\section{Measures}

Measures will be administered at the screening phase, the assessment phase (Time 1), the post intervention phase (Time 2) and post relapse prevention (Time 3). See Table 3 for an indication of the measures to be administered at each time frame.

(Insert Table 3 here)

\section{Background measures}

Cognitive function will be assessed, for the purposes of descriptive information, using the Repeatable Battery for the Assessment of Neuropsychological Status (RBANS) (Randolph, 1998). The RBANS is a brief neurocognitive battery measuring immediate and delayed memory, attention, language, and visuospatial skills and has been found to be suitable for assessing cognitive function after TBI (McKay, Wertheimer, Fichtenberg, \& Casey, 2008). The RBANS requires approximately 25 minutes to administer and is broadly used for clinical diagnostic purposes to establish neurocognitive status. Importantly it is not a measure used commonly at Liverpool BIRU and therefore removes the risk of confounding neuropsychological assessments conducted by the service. 


\section{Psychological process measures}

These measures assess the hypothesised mediator of change in the intervention. That is, they are the psychological variables that are targeted by ACT and are hypothesised to lead to improved mental health and behavioural functioning. We will examine three process variables: Psychological flexibility, participation in rehabilitation and values-consistent living.

Psychological Flexibility. The Acceptance and Action Questionnaire - Acquired Brain Injury (AAQ-ABI; Sylvester, 2011) will be used to measure psychological flexibility. The AAQ-ABI is a 15-item questionnaire assessing both acceptance and avoidance of thoughts that may arise as a result of a brain injury and was used in a study with paediatric acquired brain injury (Sylvester, 2011). For the present study the wording of one item receives a minor revision to clarify its meaning further (item 5 "My brain injury defines me” to "My brain injury defines me as a person”). The AAQ-ABI uses a 5-point Likert scale (0='not at all true' to 4=’very true') with scores ranging from 0 to 60 and higher scores indicative of greater acceptance. Initial analysis demonstrates it correlates highly with the Acceptance and Action Questionnaire-II (AAQ-II) $\left(r_{\mathrm{s}}=.61, \mathrm{~N}=66, p<\right.$ .000) (Whiting; unpublished data). As the AAQ-ABI has yet to be fully validated, the more commonly used measure of psychological flexibility in ACT research, the AAQ-II (Bond et al., 2011) will also be administered. The AAQ-II is a 10-item questionnaire utilising a 7-point Likert scale with scores ranging from 0 to 70 , with higher scores indicative of greater psychological flexibility or acceptance. Higher scores on the AAQ-II are associated with lower scores of psychological distress and it has satisfactory structure, reliability and validity have been demonstrated across a number of samples (Chronbach's alpha ranging from 0.78 to 0.88 ). The main difference between the AAQ-ABI and the AAQ-II relates to specific reference to brain injury in the items. 
Participation in rehabilitation. The Motivation for Traumatic Brain Injury Rehabilitation Questionnaire (MOT-Q) (Chervinsky et al., 1998) will be used to measure intent to engage in rehabilitation. The MOT-Q is a 31-item questionnaire with Likert-type response format with three subscales assessing attitudes to brain injury rehabilitation. The measure will be used to determine whether there is a change in participant's willingness to engage in the rehabilitation process. The scale has four subscales, Lack of Denial, Interest in Rehabilitation, Lack of Anger and Reliance on Professional Help. Reliability assessed by Cronbach's Alpha was 0.91 for the total scale (Chervinsky et al., 1998).

Values-consistent living. The Survey of Life Principles Version 2.2 - Card sorting task (CST) (Ciarrochi \& Bailey, 2008) will be administered to assist in identifying the top ten values. A measure of commitment to values was created (see Appendix I) and this will be used to measure committed action in accordance with each participants identified values. The CST will also be used as a therapeutic tool during the ACT treatment condition.

\section{Secondary Outcome Measures}

Psychological distress. The Hospital Anxiety and Depression Scale (HADS) (Zigmond \& Snaith, 1983) will be administered to assess psychological distress in part because it tends to be less confounded by somatic symptoms when compared to other anxiety and depression scales and has been found to sensitive to changes in a TBI population (Draper, Ponsford, \& Schönberger, 2007). The HADS has two subscales (7-items each) measuring self-reported anxiety and depression with score ranges of $0-21$. The subscales have high internal consistency (Chronbach $\alpha=0.90$, Moorey et al., 1991) and high test-retest reliability ( $r=0.92$, Zigmond \& Snaith, 1983). The Positive and Negative Affect Scales (PANAS) (Watson, Clark, \& Tellegen, 1988) will also be used for emotional distress but in addition will provide a measure of positive affect. The PANAS is a 20 
item measure with 10 words relating to positive mood states and 10 words relating to negative mood. Respondents are asked to rate on a 5-point scale, the extent they would normally feel that emotion (where 1 = never and 5 = always). The PANAS has good internal consistency on both subscales and is sensitive to short term mood states (Watson et al., 1988).

Weekly administered measures of psychological distress will include the Depression Anxiety Stress Scale-21 (DASS-21) (Lovibond \& Lovibond, 1995) and the Positive and Negative Affect Schedule - Short Form (I-PANAS-SF) (Thompson, 2007). The DASS-21 is a 21 item self-report measure that assesses depression, anxiety and stress over the previous week using a 4-point scale. It has been found to be sensitive to psychological distress in an acquired brain injury (ABI) population (Ownsworth, Little, Turner, Hawkes, \& Shum, 2008). The DASS-21 has good reliability on all three subscales (Chronbach's $\alpha=0.73-0.81$, Lovibond \& Lovibond, 1995). The I-PANASSF, is a shorter form of the PANAS and employs five words in each scale. This will be used for the weekly measures in order to reduce the burden on participants. The shortened version of the scale still retains good internal consistency (Chronbach's $\alpha=0.78 \& 0.76$, Thompson, 2007).

Social participation. The Sydney Psychosocial Reintegration Scale-2 (SPRS-2) will assess social participation. The SPRS-2 comprises 12 items rated, by either staff from the BIRU involved in the participant's rehabilitation or a family member. Each item is rated on a 5-point Likert type scale and in addition to a global score the measure is organised into three domains of psychosocial outcome (occupation, relationships, independent living). Scores range from 0 to 48 with higher scores indicating an increasing level of independence. Internal consistency, test-retest and interrater reliability and concurrent validity were all strong (Tate, Simpson, Soo, \& Lane-Brown, 2011).

Quality of life. The Short Form Health Survey (SF-12) (Ware Jr, Kosinski, \& Keller, 1996) is a 12item self-report questionnaire designed to measure a person's perceived health status across eight health concepts, including both mental and physical health. It demonstrates good reliability and 
validity (Ware Jr et al., 1996). The SF-12 has been used successfully with a TBI population, to assess health status, in previous large scale prevalence research (Anstey et al., 2004).

Psychiatric Screen. The General Health Questionnaire-12 (GHQ-12) (Hardy, Shapiro, Haynes, \& Rick, 1999) will be administered to screen for minor psychiatric disorders and in addition, it has been found to be sensitive to changes in psychological flexibility (Bond et al., 2011). It is a selfadministered questionnaire with a focus on psychological components of health assessing symptoms such as concentration, anxiety, depression and confidence. It uses a 4-point Likert scale with a score range of 0 to 36 and demonstrates good reliability (Chronbach's $\alpha=0.89$ ) and testretest correlation ( $r=0.73$ ) (Hardy et al., 1999).

Awareness of deficits. Poor self-awareness after a TBI has been found to lead to poorer outcomes (Ownsworth et al., 2007) and may impact on emotional distress (McBrinn et al., 2008) and decrease motivation to engage in treatment (Sherer et al., 1998). The Awareness questionnaire (AQ) (Sherer et al., 1998) will be used as a covariate in the analysis of the data. The Awareness questionnaire is a 17-item questionnaire relating to comparison of deficits pre and post injury on three domains including cognitive, behavioural/affective and motor sensory. Each item is rated on a 5-point scale giving a total score of between 17 and 85. It is administered to the person with the brain injury and a family member and discrepancy scores are calculated to determine the level of awareness. The scale demonstrates good internal consistency for both the person with the brain injury and the family member (Chronbach's $\alpha=0.88$, Sherer et al., 1998).

\section{Procedure}

Screening and Recruitment

Participants will be recruited from the clinical psychology waiting list or by referral by other members of the LBIRU community rehabilitation team. They will initially be screened to determine 
whether they meet criteria and then informed consent will be attained. Following completion of the background, primary and secondary outcome measures (Time 1), participants will be assigned to the ACT or Befriending treatment group.

\section{Random allocation}

Random allocation will be undertaken by a third party (an employee of the LBIRU not directly involved in the study) using a block randomisation procedure (2 participants per group). Allocation concealment will be ensured by assigning participants an ID number and forwarding the block of IDs to the staff member who will then allocate participants to either of the two conditions on a 1:1 ratio via a computer-generated set of random numbers.

\section{Treatment}

Treatment will commence simultaneously with one group receiving the ACT program and the second group receiving an equivalent number of sessions based on the Befriending protocol (Bendall et al., 2003). The sessions will be delivered by two clinical psychologists (one per group) with clinical experience in working with people with TBI. The psychologists will not be blind to the treatment condition. Sessions one to six will be undertaken weekly for a period of two hours at a negotiated time convenient for all participants. Weekly assessments will be undertaken, at the beginning of each session, with both groups (see Table 3).

Follow-up assessments and booster session

Primary and secondary outcome measures will be readministered to all participants at the end of the program after the completion of session six (Time 2). Participants will return after one month for a relapse prevention session (session seven) and will then be reassessed on the outcome measures (Time 3) immediately after the booster session. Staff undertaking the Time 2 and Time 3 
assessments will be blind to the treatment condition. Participants will be informed not to reveal the condition to which they were allocated to the assessor. To monitor assessor blinding, assessors will record if participants inadvertently revealed the condition that they were allocated to, and assessors will also be asked to guess the condition to which the participant was allocated. On the completion of the post treatment assessment, participants in the active control will be offered individual treatment by the clinical psychologist at the BIRU.

\section{Data Analysis}

We will analyse using IBM SPSS Statistics Version 19 and AMOS version 20. Data screening will be conducted and descriptives performed to assess the variable distributions (e.g., skewness, kurtosis). To deal with any violations of statistical assumptions, we will conduct both typical parametric analyses and nonparametric bootstrap analyses using 5,000 samples and the biascorrected percentile method (Mooney, Duval, \& Duval, 1993). We will not declare an effect to be significant unless it was significant in both analyses.

The intervention analyses will focus on two major questions: 1) What aspects of mental health do the interventions positively influence, and 2) by what processes do the interventions work. Figure 2 presents a model of the analyses. Model A represents the total effect of Intervention (X) on mental health Outcome(Y). This model is tested by using repeated measures analyses that assess the extent that the ACT versus Befriending intervention influences well-being at T2 and T3, relative to baseline.

Model B represents the direct effect of X on Y, and the indirect effect through the mediator (M), or psychological process variable (psychological flexibility, value success). This tests our key hypothesis that the intervention leads to improvement in mental health by increasing scores on the mediator (e.g., increasing psychological flexibility or value consistent living). Model C is a multiple mediation model, and will allow us to test the extent that our intervention unique alters 
each of our process variables and the extent that these process variables in turn uniquely influence outcomes. We will use the bootstrapping method described by Preacher and Hayes (2008) to test the meditational models. In order to establish temporal precedence (e.g., that the mediator changes before the outcome), we will focus on the influence of change in the mediators (baseline to postintervention) on changes in the outcomes (post-intervention to follow-up).

(Insert Figure 2 here)

Baseline data will be analysed to confirm that the two conditions have similar demographic and clinical characteristics. We will conduct both completer analysis (those who complete all three time points) and intention to treat analyses (Hollis \& Campbell, 1999). We will utilise the full information maximum likelihood (FIML) method to deal with missing data. This method is often preferred to other methods on both theoretical grounds (e.g. it makes less restrictive assumptions) and empirical grounds (the method appears to work better than its alternatives) (Bentler, 2006; Enders \& Bandalos, 2001).

\section{Discussion}

After sustaining a TBI, people can experience a wide range of psychological issues which impact on their ability to engage in rehabilitation and return to pre injury functioning. Current studies into the treatment of these psychological issues are limited and often do not meet guidelines for gold standard clinical trials. There is strong need for empirically validated outcome studies to understand the efficacy of effective treatment of psychological distress in a TBI population. Empirical outcome research using ACT is still in its infancy but so far has yielded positive results. However, its efficacy with people experiencing a TBI needs to be determined. There is also an increasing emphasis on the need to clarify the mechanisms by which psychological therapies produce change and an understanding of these components. 
This study will implement a RCT comparing ACT with an active control (Befriending) and strive to clarify the process of change during an ACT therapeutic intervention. In addition, the project will explore the therapeutic processes involved in ACT in an attempt to understand the relationships between psychological flexibility, participation and psychological distress after a severe TBI. The results from this study will augment the existing literature on the treatment of psychological adjustment after a severe TBI and contribute to the evidence base of therapeutic interventions for this population.

\section{Progress}

The clinical trial commenced in September 2011 and to date (November 2012) 16 people with a TBI have been screened for the program with 11 meeting the selection criteria. Three declined to participate in the research program, with one electing to engage in individual sessions with the clinical psychologist instead. This has resulted in two groups undertaking the treatment program (N=8) with 2 non-completers. Recruitment is currently underway for the third group. It is anticipated to continue recruiting for the study until December 2013 and then data analysis will be undertaken with the sample achieved at that time (projected $\mathrm{N}=24$ ). 


\section{References}

Anson, K., \& Ponsford, J. (2006). Evaluation of a coping skills group following traumatic brain injury. Brain Injury, 20(2), 167-178.

Anstey, K. J., Butterworth, P., Jorm, A. F., Christensen, H., Rodgers, B., \& Windsor, T. D. (2004). A population survey found an association between self-reports of traumatic brain injury and increased psychiatric symptoms. Journal of Clinical Epidemiology, 57(11), 1202-1209.

Baguley, I. J., Cooper, J., \& Felmingham, K. (2006). Aggressive behavior following traumatic brain injury: How common is common? The Journal of Head Trauma Rehabilitation, 21(1), 45-56.

Bendall, S., Jackson, H. J., Killackey, E., Allott, K., Johnson, T., Harrigan, S., . . McGorry, P. D. (2006). The credibility and acceptability of befriending as a control therapy in a randomized controlled trial of cognitive behaviour therapy for acute first episode psychosis. Behavioural and Cognitive Psychotherapy, 34(03), 277-291.

Bendall, S., Killackey, E., Jackson, H., \& Gleeson, J. (2003). Befriending Manual: OXYGEN Research Centre.

Bentler, P. M. (2006). EQS 6 Structural Equations Program Manual. Encino, CA: Multivariate Software, Inc.

Blanchet, S., Paradis-Giroux, A. A., Pépin, M., \& Mckerral, M. (2009). Impact of divided attention during verbal learning in young adults following mild traumatic brain injury. Brain Injury, 23(2), 111-122.

Bond, F. W., Hayes, S. C., Baer, R. A., Carpenter, K. C., Guenole, N., Orcutt, H. K., . . Zettle, R. D. (2011). Preliminary psychometric properties of the Acceptance and Action Questionnaire-II: A revised measure of psychological flexibility and acceptance. Behavior Therapy, 42, 676-688.

Borrelli, B. (2011). The assessment, monitoring, and enhancement of treatment fidelity In public health clinical trials. Journal of Public Health Dentistry, 71, S52-S63.

Brown, F. J., \& Hooper, S. (2009). Acceptance and Commitment Therapy (ACT) with a learning disabled young person experiencing anxious and obsessive thoughts. Journal of Intellectual Disabilities, 13(3), 195-201.

Brown, M., Gordon, W. A., \& Spielman, L. (2003). Participation in social and recreational activity in the community by individuals with traumatic brain injury. Rehabilitation Psychology, 48(4), 266-274.

Bryant, R. A., Moulds, M., Guthrie, R., \& Nixon, R. D. V. (2003). Treating acute stress disorder following mild traumatic brain injury. American Journal of Psychiatry, 160(3), 585-587.

Carney, N., Chesnut, R. M., Maynard, H., Mann, N. C., Patterson, P., \& Helfand, M. (1999). Effect of cognitive rehabilitation on outcomes for persons with traumatic brain injury: A systematic review. The Journal of Head Trauma Rehabilitation, 14(3), 277.

Carroll, E., \& Coetzer, R. (2011). Identity, grief and self-awareness after traumatic brain injury. Neuropsychological rehabilitation, 21(3), 289-305.

Cattelani, R., Zettin, M., \& Zoccolotti, P. (2010). Rehabilitation treatments for adults with behavioral and psychosocial disorders following acquired brain injury: A systematic review. Neuropsychology review, 20(1), 52-85.

Chawla, N., \& Ostafin, B. (2007). Experiential avoidance as a functional dimensional approach to psychopathology: An empirical review. Journal of Clinical Psychology, 63(9), 871-890.

Chervinsky, A. B., Ommaya, A. K., deJonge, M., Spector, J., Schwab, K., \& Salazar, A. M. (1998). Motivation for Traumatic Brain Injury Rehabilitation Questionnaire (MOT-Q): Reliability, 
factor Analysis, and relationship to MMPI-2 variables. Archives of Clinical Neuropsychology, 13(5), 433-446.

Dahl, J., Wilson, K. G., \& Nilsson, A. (2004). Acceptance and Commitment Therapy and the Treatment of Persons at Risk for Long-Term Disability Resulting From Stress and Pain Symptoms: A Preliminary Randomized Trial. Behavior Therapy, 35(4), 785-801.

deGuise, E., LeBlanc, J., Feyz, M., Meyer, K., Duplantie, J., Thomas, H., . . . Lin, H. (2008). Longterm outcome after severe traumatic brain injury: The McGill interdisciplinary prospective study. The Journal of Head Trauma Rehabilitation, 23(5), 294-303.

Draper, K., Ponsford, J., \& Schönberger, M. (2007). Psychosocial and emotional outcomes 10 years following traumatic brain injury. The Journal of Head Trauma Rehabilitation, 22(5), 278.

Enders, C. K., \& Bandalos, D. L. (2001). The relative performance of full information maximum likelihood estimation for missing data in structural equation models. Structural Equation Modeling, 8(3), 430-457.

Fann, J. R., Hart, T., \& Schomer, K. G. (2009). Treatment for depression after traumatic brain injury: a systematic review. Journal of Neurotrauma, 26(12), 2383-2402.

Feeley, N., Cossette, S., Cote, J., Heon, M., Stremler, R., Martorella, G., \& Purden, M. (2009). The importance of piloting an RCT intervention. CJNR (Canadian Journal of Nursing Research), 41(2), 84-99.

Fleming, J., Braithwaite, H., Gustafsson, L., Griffin, J., Collier, A. M., \& Fletcher, S. (2011). Participation in leisure activities during brain injury rehabilitation. Brain Injury(0), 1-13.

Gregg, J. A., Callaghan, G. M., Hayes, S. C., \& Glenn-Lawson, J. L. (2007). Improving diabetes self-management through acceptance, mindfulness, and values: A randomized controlled trial. Journal of Consulting and Clinical Psychology, 75(2), 336-343.

Guillamondegui, O. D., Montgomery, S. A., Phibbs, F. T., McPheeters, M. L., Alexander, P. T., Jerome, R. N., . . . Krishnaswami, S. (2011). Traumatic Brain Injury and Depression. Comparative Effectiveness Review No. 25. Rockville, MD: Vanderbilt Evidence-based Practice Center under Contract No. 290-2007-10065-I

Hardy, G. E., Shapiro, D. A., Haynes, C. E., \& Rick, J. E. (1999). Validation of the General Health Questionnaire-12: Using a sample of employees from England's health care services. Psychological Assessment, 11(2), 159-165.

Hart, P. D. T., Fann, J. R., \& Novack, T. A. (2008). The dilemma of the control condition in experience-based cognitive and behavioural treatment research. Neuropsychological Rehabilitation, 18(1), 1-21.

Hayes, S. C., \& Strosahl, K. D. (Eds.). (2004). A practical guide to Acceptance and Commitment Therapy. New York: Springer-Verlag.

Hayes, S. C., Strosahl, K. D., \& Wilson, K. G. (2003). Acceptance and commitment therapy: An experiential approach to behavior change. New York: The Guilford Press.

Heled, E., Hoofien, D., Margalit, D., Natovich, R., \& Agranov, E. (2012). The Delis-Kaplan Executive Function System Sorting Test as an evaluative tool for executive functions after severe traumatic brain injury: A comparative study. Journal of Clinical and Experimental Neuropsychology, 34(2), 151-159.

Hesser, H., Westin, V., Hayes, S. C., \& Andersson, G. (2009). Clients' in-session acceptance and cognitive defusion behaviors in acceptance-based treatment of tinnitus distress. Behaviour Research and Therapy, 47(6), 523-528.

Hibbard, M. R., Rendon, D., Charatz, H., \& Kothera, L. (2005). CBT in individuals with traumatic brain injury. Cognitive behavior therapy in nursing practice, 189.

Hibbard, M. R., Uysal, S., Kepler, K., Bogdany, J., \& Silver, J. (1998). Axis I psychopathology in individuals with traumatic brain injury. The Journal of Head Trauma Rehabilitation. 
Hodgson, J., McDonald, S., Tate, R., \& Gertler, P. (2005). A randomised controlled trial of a cognitive-behavioural therapy program for managing social anxiety after acquired brain injury. Brain Impairment, 6(3), 169-180.

Hoffman, S. W., \& Harrison, C. (2009). The interaction between psychological health and traumatic brain injury: a neuroscience perspective. The Clinical Neuropsychologist, 23(8), 1400-1415.

Hollis, S., \& Campbell, F. (1999). What is meant by intention to treat analysis? Survey of published randomised controlled trials. British Medical Journal, 319(7211), 670-674.

Hsieh, M. Y., Ponsford, J., Wong, D., Schonberger, M., McKay, A., \& Haines, K. (2012). A cognitive behaviour therapy (CBT) programme for anxiety following moderatesevere traumatic brain injury (TBI): Two case studies. Brain Injury, 26(2), 126-138.

Hsieh, M. Y., Ponsford, J., Wong, D., Schönberger, M., Taffe, J., \& Mckay, A. (2012). Motivational interviewing and cognitive behaviour therapy for anxiety following traumatic brain injury: A pilot randomised controlled trial. Neuropsychological rehabilitation, 22(4), 585-608.

Kangas, M., \& McDonald, S. (2011). Is it time to act? The potential of acceptance and commitment therapy for psychological problems following acquired brain injury. Neuropsychological Rehabilitation, 21(2), 250-276.

Kashdan, T. B., \& Rottenberg, J. (2010). Psychological flexibility as a fundamental aspect of health. Clinical Psychology Review, 30(4), 467-480.

Khan-Bourne, N., \& Brown, R. (2003). Cognitive behaviour therapy for the treatment of depression in individuals with brain injury. Neuropsychological rehabilitation, 13(1-2), 89-107.

Kinney, A. (2001). Cognitive therapy and brain-injury: Theoretical and clinical issues. Journal of Contemporary Psychotherapy, 31(2), 89-102.

Lovibond, S., \& Lovibond, P. (1995). Manual for the Depression Anxiety Stress Scales (2nd ed.). Sydney: Psychology Foundation.

McBrinn, J., Colin Wilson, F., Caldwell, S., Carton, S., Delargy, M., McCann, J., . . McGuire, B. (2008). Emotional distress and awareness following acquired brain injury: An exploratory analysis. Brain Injury, 22(10), 765-772.

McKay, C., Wertheimer, J. C., Fichtenberg, N. L., \& Casey, J. E. (2008). The Repeatable Battery for the Assessment of Neuropsychological Status (RBANS): Clinical utility in a traumatic brain injury sample. The Clinical Neuropsychologist, 22(2), 228-241.

Mead, N., Lester, H., Chew-Graham, C., Gask, L., \& Bower, P. (2010). Effects of befriending on depressive symptoms and distress: systematic review and meta-analysis. The British Journal of Psychiatry, 196(2), 96-101.

Medd, J., \& Tate, R. L. (2000). Evaluation of an anger management therapy programme following acquired brain injury: A preliminary study. Neuropsychological Rehabilitation, 10(2), 185201.

Mooney, C. Z., Duval, R. D., \& Duval, R. (1993). Bootstrapping: A nonparametric approach to statistical inference: Sage Publications, Inc.

Moorey, S., Greer, S., Watson, M., Gorman, C., Rowden, L., Tunmore, R., . . . Bliss, J. (1991). The factor structure and factor stability of the hospital anxiety and depression scale in patients with cancer. The British Journal of Psychiatry, 158(2), 255-259.

Myles, S. M. (2004). Understanding and treating loss of sense of self following brain injury: A behavior analytic approach. International Journal of Psychology and Psychological Therapy, 4(3), 487-504.

Ownsworth, T., Fleming, J., Shum, D., Kuipers, P., \& Strong, J. (2008). Comparison of individual, group and combined intervention formats in a randomized controlled trial for facilitating goal attainment and improving psychosocial function following acquired brain injury. Journal of Rehabilitation Medicine, 40(2), 81-88. 
Ownsworth, T., Fleming, J., Strong, J., Radel, M., Chan, W., \& Clare, L. (2007). Awareness typologies, long-term emotional adjustment and psychosocial outcomes following acquired brain injury. Neuropsychological Rehabilitation, 17(2), 129-150.

Ownsworth, T., Little, T., Turner, B., Hawkes, A., \& Shum, D. (2008). Assessing emotional status following acquired brain injury: The clinical potential of the depression, anxiety and stress scales. Brain Injury, 22(11), 858-869.

Ponsford, J., Sloan, S., \& Snow, P. (1995). Traumatic brain injury: Rehabilitation for everyday adaptive living. Hillsdale, NJ, England: Lawrence Erlbaum Associates, Inc.

Preacher, K. J., \& Hayes, A. F. (2008). Asymptotic and resampling strategies for assessing and comparing indirect effects in multiple mediator models. Behavior research methods, 40(3), 879-891.

Randolph, C. (1998). RBANS manual: Repeatable Battery for the Assessment of Neuropsychological Status. San Antonio, TX: The Psychological Corporation.

Rao, V., Rosenberg, P., Bertrand, M., Salehinia, S., Spiro, J., Vaishnavi, S., . . . Brandt, J. (2009). Aggression after traumatic brain injury: Prevalence \& correlates. The Journal of Neuropsychiatry and Clinical Neurosciences, 21(4), 420-429.

Ruiz, F. J. (2010). A review of Acceptance and Commitment Therapy (ACT) empirical evidence: Correlational, experimental psychopathology, component and outcome studies. International Journal of Psychology and Psychological Therapy(1), 125-162.

Russell, W., \& Smith, A. (1961). Post-traumatic amnesia in closed head injury. Archives of Neurology, 5(1), 4-17.

Schlund, M. W., \& Pace, G. (1999). Relations between traumatic brain injury and the environment: feedback reduces maladaptive behaviour exhibited by three persons with traumatic brain injury. Brain Injury, 13(11), 889-897.

Schulz, K., Altman, D., \& Moher, D. (2010). CONSORT 2010 Statement: Updated guidelines for reporting parallel group randomised trials. British Medical Journal, 340, 698-702.

Sherer, M., Boake, C., Levin, E., Silver, B. V., Ringholz, G., \& High, W. M. (1998). Characteristics of impaired awareness after traumatic brain injury. Journal of the International Neuropsychological Society, 4(04), 380-387.

Simpson, G. K., Tate, R. L., Whiting, D. L., \& Cotter, R. E. (2011). Suicide prevention after traumatic brain injury: A randomized controlled trial of a program for the psychological treatment of hopelessness. The Journal of Head Trauma Rehabilitation, 26(4), 290-300.

Soo, C., \& Tate, R. (2009). Psychological treatment for anxiety in people with traumatic brain injury. Cochrane Database of Systematic Reviews, 3, Available at: http://www.thecochranelibrary.com/.

Soo, C., Tate, R. L., \& Lane-Brown, A. (2011). A systematic review of acceptance and commitment therapy (ACT) for managing anxiety: Applicability for people with acquired brain injury? Brain Impairment, 12(1), 54-70.

Sylvester, M. (2011). Acceptance and commitment therapy for improving adaptive functioning in persons with a history of pediatric acquired brain injury. Doctor of Philosophy in Clinical Psychology Dissertation, University of Nevada, Reno.

Tate, R. L., \& Douglas, J. (2011). Use of reporting guidelines in scientific writing: PRISMA, CONSORT, STROBE, STARD and other resources. Brain Impairment, 12(1), 1-21.

Tate, R. L., Simpson, G. K., Soo, C. A., \& Lane-Brown, A. T. (2011). Participation after acquired brain injury: Clinical and psychometric considerations of the Sydney Psychosocial Reintegration Scale (SPRS). Journal of Rehabilitation Medicine, 43(7), 609-618.

Thompson, E. R. (2007). Development and validation of an internationally reliable short-form of the positive and negative affect schedule (PANAS). Journal of Cross-Cultural Psychology, 38(2), 227-242. 
Veehof, M. M., Oskam, M. J., Schreurs, K. M. G., \& Bohlmeijer, E. T. (2011). Acceptance-based interventions for the treatment of chronic pain: A systematic review and meta-analysis. Pain, 152, 533-542.

Ware Jr, J. E., Kosinski, M., \& Keller, S. D. (1996). A 12-Item Short-Form Health Survey: Construction of scales and preliminary tests of reliability and validity. Medical Care, 34(3), 220-233.

Whitehouse, A. M. (1994). Applications of cognitive therapy with survivors of head injury. Journal of Cognitive Psychotherapy, 8(2), 141-160.

Whiting, D., Simpson, G., Ciarrochi, J., \& McLeod, H. (2012). Assessing the feasibility of Acceptance and Commitment Therapy in promoting psychological adjustment after severe traumatic brain injury. Brain Injury, 26(4-5), 588-589.

Whyte, J., \& Hart, T. (2003). It's more than a black box; it's a Russian doll: Defining rehabilitation treatments. American Journal of Physical Medicine \& Rehabilitation, 82(8), 639-652.

Wood, R. L., \& Alderman, N. (2011). Applications of operant learning theory to the management of challenging behavior after traumatic brain injury. The Journal of Head Trauma Rehabilitation, 26(3), 202-211.

Ylvisaker, M., Turkstra, L., Coehlo, C., Yorkston, K., Kennedy, M., Sohlberg, M. K. M., \& Avery, J. (2007). Behavioural interventions for children and adults with behaviour disorders after TBI: A systematic review of the evidence. Brain Injury, 21(8), 769-805.

Zigmond, A. S., \& Snaith, R. (1983). The Hospital Anxiety and Depression Scale. Acta psychiatrica scandinavica, 67(6), 361-370. 


\section{Values Card Sort}

Participant Number:

Date:

Instructions:

Ask the participant to sort the cards into three piles.

1. Pile 1: These values are not very important to me

2. Pile 2: These values are of moderate importance to me

3. Pile 3: These values are of the highest importance to me

4. Discard pile 1 , shuffle piles $2 \& 3$ and repeat steps $1-3$

5. From Pile 3 (the highest importance), have the clients identify their top 10 values

6. Rank them in order

7. Rate how important that value is in your life at the present time

8. Rate how well you are acting in accordance to that value

\begin{tabular}{|c|c|c|c|c|c|c|c|c|}
\hline \multicolumn{9}{|c|}{ Value 1: } \\
\hline \multicolumn{5}{|c|}{ How important is this value? } & \multicolumn{4}{|c|}{ How consistently are you acting in accordance with your value? } \\
\hline 0 & 1 & 2 & 3 & 4 & 1 & 2 & 3 & 4 \\
\hline \multicolumn{9}{|c|}{ Value 2: } \\
\hline \multicolumn{5}{|c|}{ How important is this value? } & \multicolumn{4}{|c|}{ How consistently are you acting in accordance with your value? } \\
\hline 0 & 1 & 2 & 3 & 4 & 1 & 2 & 3 & 4 \\
\hline \multicolumn{9}{|c|}{ Value $3:$} \\
\hline \multicolumn{5}{|c|}{ How important is this value? } & \multicolumn{4}{|c|}{ How consistently are you acting in accordance with your value? } \\
\hline 0 & 1 & 2 & 3 & 4 & 1 & 2 & 3 & 4 \\
\hline
\end{tabular}

Note: For the purposes of illustration only part of the questionnaire is reproduced here. The full questionnaire rates the top 10 values. 
Table 3: Administration of Measures -Intervention Study

\begin{tabular}{|c|c|c|c|c|}
\hline Measure & $\begin{array}{l}\text { Baseline } \\
\text { (T1) }\end{array}$ & $\begin{array}{c}\text { Weekly } \\
\text { measures }\end{array}$ & $\begin{array}{c}\text { Post-intervention } \\
\text { (T2) }\end{array}$ & $\begin{array}{l}\text { Follow-up } \\
\text { (T3) }\end{array}$ \\
\hline \multicolumn{5}{|l|}{ Participant } \\
\hline RBANS & $\mathrm{x}$ & & & \\
\hline Demographic & $\mathrm{x}$ & & & \\
\hline \multicolumn{5}{|l|}{ information } \\
\hline AAQ-ABI & $\mathrm{x}$ & $\mathrm{x}$ & $x$ & $\mathrm{x}$ \\
\hline HADS & $\mathrm{x}$ & & $\mathrm{x}$ & $\mathrm{x}$ \\
\hline DASS-21 & $\mathrm{x}$ & $\mathrm{x}$ & $\mathrm{x}$ & $\mathrm{x}$ \\
\hline GHQ-12 & $\mathrm{x}$ & & $\mathrm{x}$ & $\mathrm{x}$ \\
\hline PANAS & $\mathrm{x}$ & & $\mathrm{x}$ & $\mathrm{x}$ \\
\hline AAQ-II & $\mathrm{x}$ & $\mathrm{x}$ & $\mathrm{x}$ & $\mathrm{x}$ \\
\hline MOT-Q & $\mathrm{x}$ & & $\mathrm{x}$ & $\mathrm{x}$ \\
\hline $\mathrm{AQ}$ & $\mathrm{x}$ & & $\mathrm{x}$ & $\mathrm{x}$ \\
\hline CST & $\mathrm{x}$ & & $\mathrm{x}$ & $\mathrm{x}$ \\
\hline I-PANAS-SF & & $\mathrm{x}$ & & \\
\hline \multicolumn{5}{|c|}{ Family Member } \\
\hline $\mathrm{AQ}$ & $\mathrm{x}$ & & $\mathrm{x}$ & $\mathrm{x}$ \\
\hline SPRS-2 & $\mathrm{x}$ & & $\mathrm{x}$ & $\mathrm{x}$ \\
\hline \multicolumn{5}{|c|}{$\begin{array}{l}\text { RBANS: Repeatable Battery for the Assessment of Neuropsychological Status, AAQ-ABI: Acceptance and Action } \\
\text { Questionnaire - Acquired Brain Injury, HADS: Hospital Anxiety and Depression Scale, DASS-21: Depression Anxiety } \\
\text { Stress Scale-21, GHQ-12: General Health Questionnaire-12, PANAS: Positive Affect Negative Affect Scale, AAQ-II: } \\
\text { Acceptance and Action Questionnaire-II, MOT-Q: Motivation for Traumatic Brain Injury Rehabilitation Questionnaire, } \\
\text { AQ: Awareness Questionnaire, CST: Survey of Life Principles - Card Sort Task, I-PANAS-SF: Positive and Negative } \\
\text { Affect Schedule - Short Form, SPRS-2: Sydney Psychosocial Reintegration Scale-2. }\end{array}$} \\
\hline
\end{tabular}




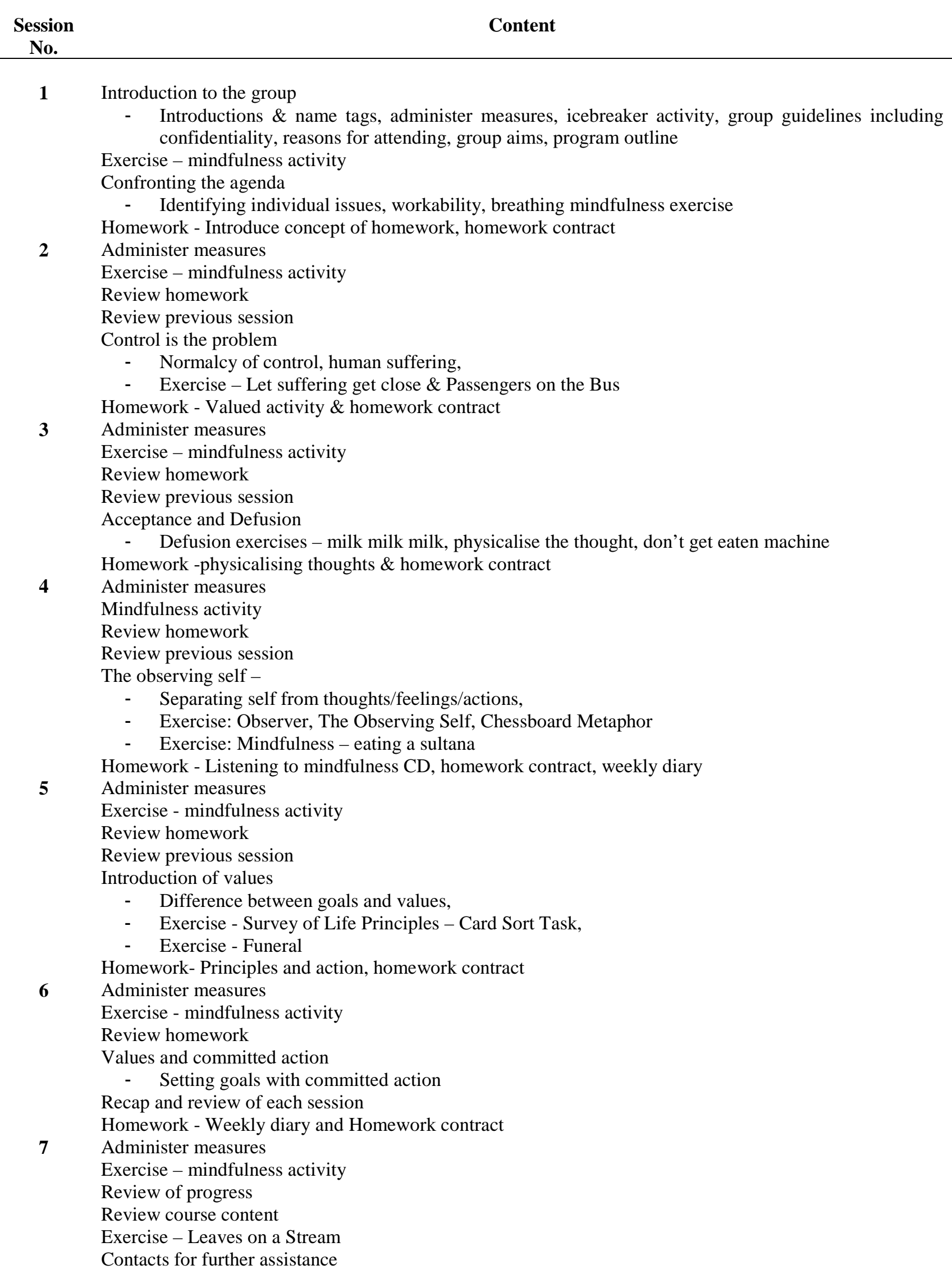


Table 2: Summary of Befriending (active control) program

Session

\section{Content}

1

Discussion of group rules and aims

Introduction of each group member

Activity - Identification of what we will cover each week

Suggested Activities

- Going for a coffee

- Watch a movie over the week and discuss next session

- Teach the other members to play a card game

- Each person in the group speak about their favourite hobby/activity

- $\quad$ Brain Storm other ideas

Set timetable for activities for future sessions

2 - 6 Session content set according to timetable established in Session 1

$7 \quad$ Review of progress over previous month

Discussion of referral for ongoing services 